\title{
Effects of continuous positive airway pressure on coagulability in obstructive sleep apnoea: a randomised, placebo-controlled crossover study
}

\author{
Craig L Phillips, ${ }^{1,2}$ Bradley J McEwen, ${ }^{3,4}$ Marie-Christine Morel-Kopp, ${ }^{3,4}$ \\ Brendon J Yee, ${ }^{2,5}$ David R Sullivan, ${ }^{6}$ Christopher M Ward, ${ }^{3,4}$ Geoffrey H Tofler, \\ Ronald R Grunstein ${ }^{2,5}$
}

\begin{abstract}
'Department of Respiratory and Sleep Medicine, Royal North Shore Hospital, St Leonards, New South Wales, Australia ${ }^{2}$ NHMRC Centre for Sleep Health, Woolcock Institute of Medical Research, University of Sydney, Sydney, New South Wales, Australia

${ }^{3}$ Northern Blood Research Centre, Kolling Institute of Medical Research, University of Sydney, Sydney, New South Wales, Australia

${ }^{4}$ Department of Haematology and Transfusion Medicine, Royal North Shore Hospital, St Leonards, New South Wales, Australia

${ }^{5}$ Department of Respiratory and Sleep Medicine, Royal Prince Alfred Hospital, Camperdown, New South Wales, Australia ${ }^{6}$ Department of Clinical Biochemistry, Royal Prince Alfred Hospital, St Leonards, New South Wales, Australia 7Department of Cardiology, Royal North Shore Hospital, St Leonards, New South Wales, Australia
\end{abstract}

\section{Correspondence to} Dr Craig L Phillips, Woolcock Institute of Medical Research, PO Box M77, Missenden Road, NSW 2050, Australia; craig.phillips@sydney.edu.au

CLP, BJM are equal first authors.

Received 28 July 2011 Accepted 15 January 2012 Published Online First 14 February 2012

\section{ABSTRACT}

Introduction Obstructive sleep apnoea (OSA) is associated with increased cardiovascular risk, however the mechanisms are not well established.

Objectives This study aimed to determine whether treatment of OSA with nasal continuous positive airway pressure (CPAP) would favourably alter coagulability across the sleep-wake cycle.

Methods In a randomised crossover trial, 28 patients received therapeutic or placebo CPAP, each for 2 months with a 1 month washout between treatments. After each treatment period, a $24 \mathrm{~h}$ coagulation study was conducted in the laboratory. Plasminogen activator inhibitor-1 (PAl-1), D-dimer, fibrinogen, von Willebrand Factor (VWF), factor VIII (FVIII), factor VII (FVII) and factor V (FV) were determined at seven time points over the day and night.

Results At baseline, patients had severe OSA (Apnoea Hypopnoea Index $37.9 \pm 23.9$ events/h). Treatment of OSA with CPAP compared with placebo resulted in lower $24 \mathrm{~h}$ levels of vWF $(-3.9 \%, p=0.013)$, FVIII $(-6.2 \%$, $p=0.007)$ and $F V(-4.2 \%, p<0.001)$. The greatest difference occurred during the nocturnal and early morning periods. In contrast, fibrinogen, D-dimer, FVII and PAl-1 did not differ between treatments, however all markers displayed diurnal variability independent of treatment.

Conclusions In this randomised, placebo-controlled crossover trial, treatment of OSA with CPAP reduced the early morning level of VWF, and nocturnal levels of FVIII and FV. These findings suggest that CPAP may reduce cardiovascular risk in OSA, in part through reducing risk of thrombosis.

\section{INTRODUCTION}

Obstructive sleep apnoea (OSA) is a condition characterised by obstructed breathing during sleep causing sleep fragmentation and intermittent hypoxaemia. Longitudinal cohort studies have shown that people with OSA are at increased risk of all-cause ${ }^{1}$ and cardiovascular mortality ${ }^{2}$ as well as incident stroke ${ }^{3}$ and coronary heart disease. ${ }^{4}$ Nasal continuous positive airway pressure (CPAP) is considered the gold standard treatment for OSA and long-term treatment of severe OSA has been associated with a reduction in cardiovascular events and mortality near to levels found in apparently healthy people. ${ }^{5}$ While the mechanisms responsible

\section{Key messages}

What is the key question?

- Does treatment for obstructive sleep apnoea with continuous positive airway pressure (CPAP) improve coaguability, an intermediate marker of cardiovascular risk?

What is the bottom line?

- Yes, but this is only evident for some markers.

Why read on?

- The changes in coagulation markers after CPAP treatment were particularly evident during the night, a period which has been associated with increased cardiovascular events and death in this group.

for increased cardiovascular risk in OSA and a lowering of risk with CPAP treatment are still unclear, hypertension may be a modifiable risk factor $^{6}$ that plays a role. In addition, direct improvements in vascular endothelial function and inflammatory markers have been demonstrated following CPAP treatment and these are also likely to contribute to improved outcome. ${ }^{7} 8$ Another related yet less well studied component that may contribute to increased cardiovascular risk is increased coagulability. A number of observational studies from clinic $^{9-12}$ and community ${ }^{13}$ populations have demonstrated an association between OSA and prothrombotic factors. There is also limited evidence from CPAP intervention studies showing that OSA treatment decreases some markers of coagulation. ${ }^{14-16}$ Although two of these studies included randomised placebo-controlled designs, ${ }^{14} 16$ one study was small with a short 2 week treatment period ${ }^{14}$ and neither study assessed coagulation markers across time under carefully controlled conditions.

Studies which have sampled coagulation markers over $24 \mathrm{~h}$ suggest that many display a diurnal rhythm, ${ }^{17}$ with a timing that shows increased coagulability in the early morning. This temporal pattern coincides with a peak in the occurrence of cardiovascular events such as myocardial infarction (MI) and stroke, ${ }^{17}$ which in general populations, occur more often from 06:00 until 12:00 ${ }^{18}$ with 
a peak during the first few hours of awakening ${ }^{19}$ and an increase in occurrence of sudden cardiac death between 07:00 and 09:00. ${ }^{20} 21$ This post-awakening increase in cardiac events contrasts with patients with OSA in whom the frequency of MI or sudden cardiac death is significantly higher during sleep between 00:00 and 06:00. ${ }^{20} 22$ Increased platelet aggregation and activation during the night has been found in patients with OSA compared with the pre-sleep period with reductions following CPAP treatment. ${ }^{23}$ In contrast, limited data suggest similar temporal alignment of rhythms in some fibrinolytic markers between patients with OSA and controls albeit with a reduced fibrinolytic capacity. ${ }^{10}$ Despite this evidence, there are no robustly designed CPAP intervention studies of sufficient duration that would support a causative role for increased coagulability in OSA and/or a shift in the diurnal rhythm of coagulation markers.

In this study we investigated whether coagulability during both wakefulness and sleep in patients with severe sleep apnoea improves after 2 months of treatment with CPAP using a randomised placebo-controlled crossover design.

\section{METHODS}

\section{Patients}

The protocol has previously been reported in a study which assessed the effect of CPAP treatment on postprandial lipidemia. ${ }^{24}$ Details of randomisation, allocation concealment and blinding and the flow of patients through the trial are included in the original report. ${ }^{24}$ Briefly, we recruited patients from sleep apnoea clinics who were over 21 years old with OSA severity in the upper moderate or severe range (Apnoea Hypopnoea Index $(\mathrm{AHI}) \geq 25)$ with a hypoxic component (Oxygen Desaturation Index $(\mathrm{ODI}) \geq 20$ ). We excluded patients with body mass index $(\mathrm{BMI})>35 \mathrm{~kg} / \mathrm{m}^{2}$, with uncontrolled type 2 diabetes, with liver disease and patients who had previously used CPAP. Other selection criteria are available from the Australian and New Zealand Clinical Trials Registry (ACTRN 12605000066684, available at http://www.anzctr.org.au). The diagnosis of sleep apnoea was based on full overnight polysomnography in a clinical laboratory prior to recruitment. Apnoeas and hypopneas were scored using standard scoring techniques. ${ }^{25}$

\section{Protocol}

Patients who met the inclusion/exclusion criteria were randomised to receive CPAP or sham CPAP each for 2 months in a crossover design with an intervening 1-month washout period between treatments (figure 1). Sham CPAP devices are identical in appearance to the therapeutic devices except that the pressure delivered at the mask remains at $0.5 \mathrm{~cm} \mathrm{H} \mathrm{H}_{2} \mathrm{O}$, having no effect on sleep disordered breathing. In contrast, therapeutic CPAP was set to a pressure which prevented most sleep disordered

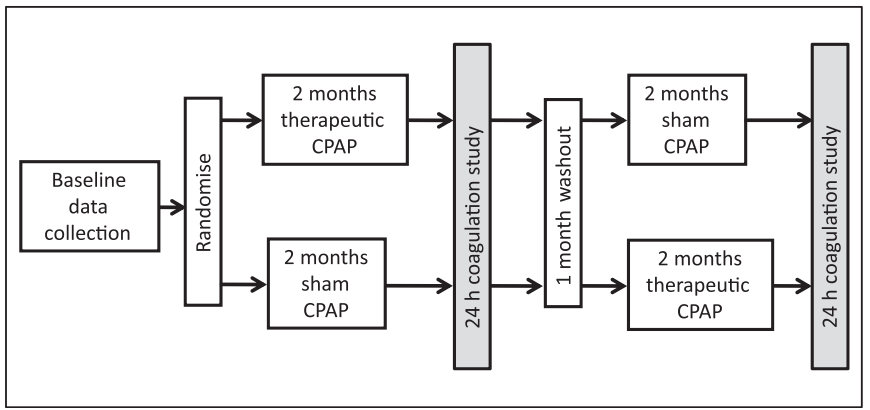

Figure 1 Study protocol. CPAP, continuous positive airway pressure. breathing. This was determined during an at home pressure determination study as previously described. ${ }^{24}$

\section{Coagulation studies}

Twenty-four hour coagulation markers (see below) were assessed at seven time points encompassing wake and sleep periods during an in-laboratory study performed at the end of each 2 -month treatment arm. Collection times were within the 30 min prior to meals at 09:00 (breakfast), 15:00 (lunch), 21:00 (dinner), and also at 00:00, 03:00, 06:00 and 09:00 h (before breakfast). The first measurement was performed immediately upon arrival at the facility (the Woolcock Institute of Medical Research, Sydney) and prior activity levels were not controlled. In contrast, the final measurement was performed following a period of relative inactivity. The measurements during the sleep period were performed by briefly awakening the patient during the night. The timing and content of daytime meals were identical during each study (figures 2 and 3). Meals were based on a western style diet as previously described. ${ }^{24}$ During the sleep period of the laboratory studies, each patient was asked to use their treatment device as they had been doing at home.

\section{Coagulation marker assays}

Blood $(4.5 \mathrm{ml})$ was drawn from an antecubital vein into tubes containing $0.129 \mathrm{M}$ tri-sodium citrate (3.2\%). Samples were then centrifuged at $3080 \mathrm{~g}$ for $5 \mathrm{~min}$ during which the platelet poor plasma component was separated and put into aliquots and then stored at $-80^{\circ} \mathrm{C}$. After storage, frozen samples were thawed and measured on a Compact Coag Analyser (Diagnostica Stago, Asnières sur Seine, France). The following parameters were measured: plasminogen activator inhibitor-1 (PAI-1), D-dimer, fibrinogen, von Willebrand factor (vWF), factor VIII (FVIII), factor VII (FVII) and factor V (FV). Fibrinogen was measured using the Clauss method. vWF antigen and D-dimer were assayed using an immunoturbidimetric method. FVIII activity was measured using an activated partial thromboplastin time clot base assay and FV and FVII activities using a prothrombin time clot base assay. PAI-1 activity was measured using a colorimetric assay. All kits and reagents were from Diagnostica Stago. To reduce inter-assay variation, all CPAP and placebo samples from a single patient were run in batches and analysed simultaneously.

\section{Statistical methods}

All coagulation markers were analysed over $24 \mathrm{~h}$ using linear mixed models. The treatment, order, time point and treatment by time point interaction were included as fixed effects and patient was a random effect in all models. The final analyses included 28 patients with usable samples who had completed the crossover trial, regardless of compliance with either therapeutic or sham CPAP. PASW statistics V.17 was used for all analyses.

\section{RESULTS}

The baseline characteristics of the patients who completed the study are shown in table 1 . Two patients had controlled type 2 diabetes and a number of patients had hypertension and/or hypercholesterolaemia. Importantly, only one patient was taking medication that influences coagulability, which was aspirin. Overall, the patients were middle aged and obese with severe OSA. Body weight did not change during the trial and no patients commenced any new medication or altered the dose or ingestion time of existing medication (including statins and anti-hypertensives). The details of patient flow through the 


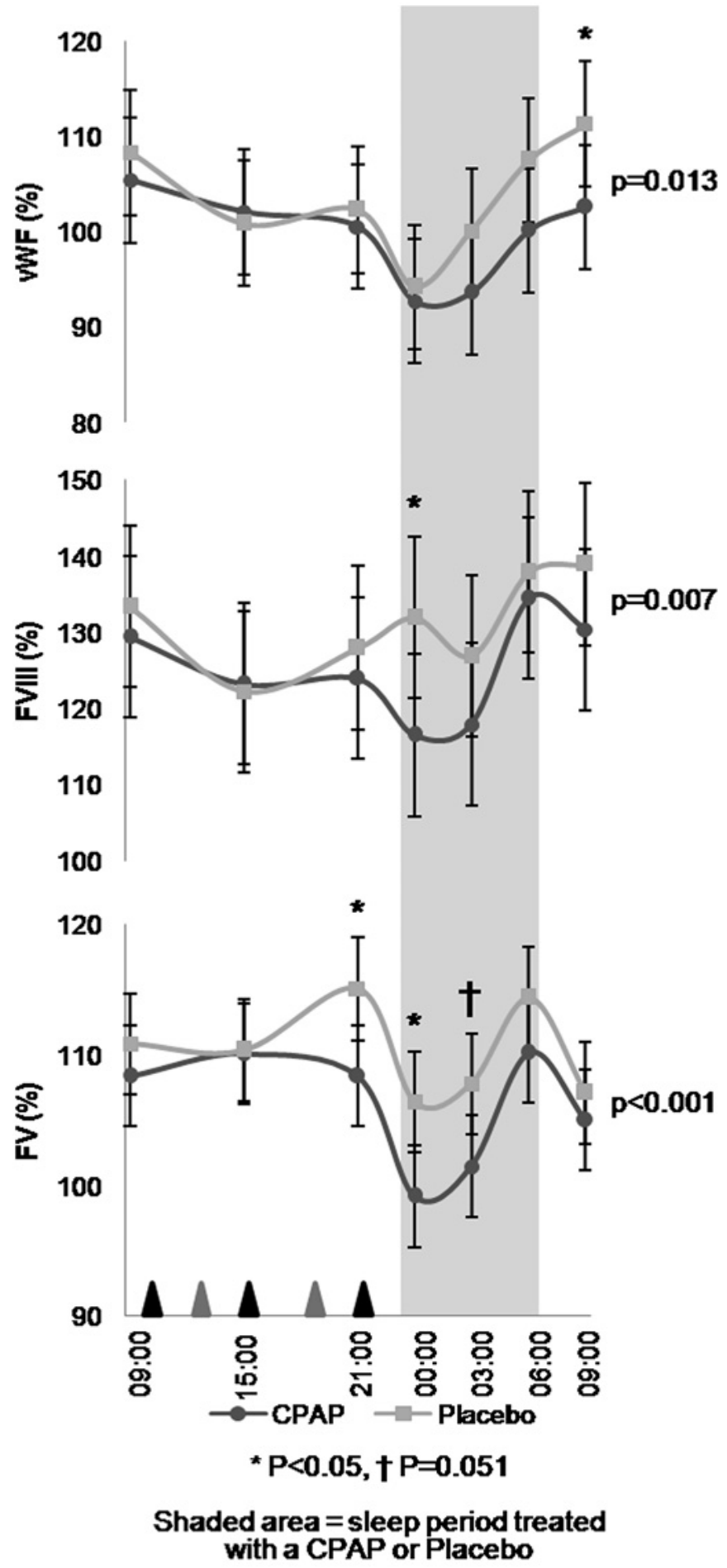

\section{Meal Snack}

Figure 2 Temporal changes in coagulation factors von Willebrand factor (VWF), factor VIII (FVIII) and factor V (FV) across the wake and sleep periods during continuous positive airway pressure (CPAP) and placebo end of treatment studies. Note sample 1 occurred soon after arrival at the laboratory whereas sample 7 occurred after restricted activity. Data are means $( \pm S E M)$. $p$ Values are for main treatment effects.

study have been previously described. ${ }^{24}$ Briefly, 120 patients were screened for eligibility with 83 being excluded, mainly for not meeting inclusion criteria. Of the 37 who were randomised,

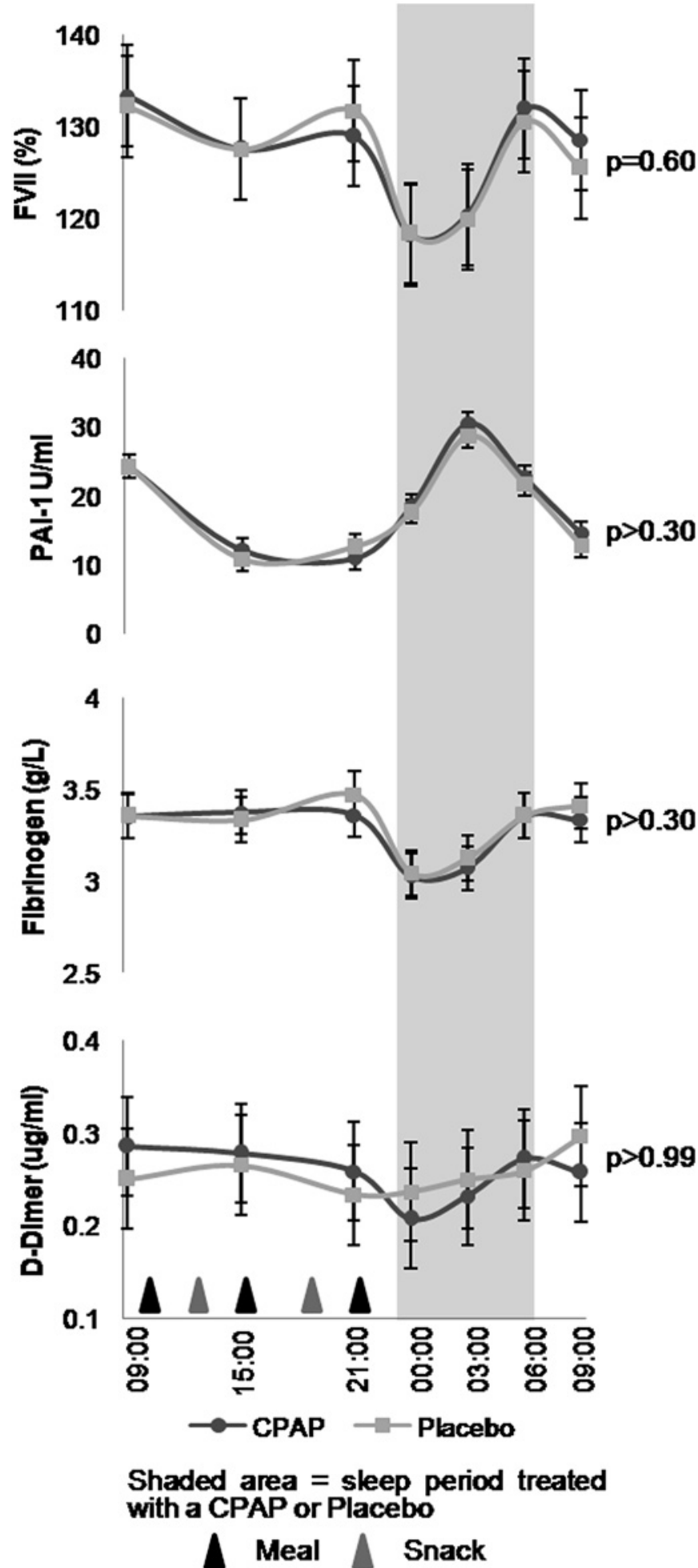

Figure 3 Temporal changes in coagulation factors plasminogen activator inhibitor-1 (PAI-1), factor (FVII), fibrinogen and D-dimer across the wake and sleep periods during continuous positive airway pressure (CPAP) and placebo end of treatment studies. Note sample 1 occurred soon after arrival at the laboratory whereas sample 7 occurred after restricted activity. Data are means $( \pm$ SEM). $p$ Values are for main treatment effects.

29 completed the study, however blood samples from one patient could not be analysed. Of the eight patients who did not complete the study, five withdrew because of adverse events 
Table 1 Patient demographic and anthropometric data

\begin{tabular}{|c|c|}
\hline Characteristic & \\
\hline \multicolumn{2}{|l|}{ Demographics } \\
\hline Age (years), mean (SD) & $49(14)$ \\
\hline Men/women, n & $25 / 3$ \\
\hline BMI $\left(\mathrm{kg} / \mathrm{m}^{2}\right)$, mean $(\mathrm{SD})$ & $31.7(4.1)$ \\
\hline \multicolumn{2}{|c|}{ Medical history and medication, $\mathrm{n}$} \\
\hline Hypertension & 9 \\
\hline Type 2 diabetes & 2 \\
\hline Hypercholesterolaemia & 10 \\
\hline Antihypertensives & 9 \\
\hline Statins & 7 \\
\hline Aspirin & 1 \\
\hline \multicolumn{2}{|l|}{ Sleep apnoea, mean (SD) } \\
\hline AHI (events/h) & $37.9(23.9)$ \\
\hline ODI (events/h) & $31.3(22.4)$ \\
\hline $\mathrm{SaO}_{2}-\mathrm{T} 90$ (\%TST) & $6.90(11.3)$ \\
\hline $\mathrm{Min} \mathrm{SaO}_{2}(\%)$ & $78.3(11.0)$ \\
\hline ESS & $10.3(4.90)$ \\
\hline
\end{tabular}

AHI, Apnoea Hypopnoea Index; BMI, body mass index; ESS, Epworth sleepiness score; $\mathrm{Min} \mathrm{SaO}_{2}$, minimum arterial oxygen saturation; $\mathrm{ODI}$ Oxygen Desaturation Index; $\mathrm{SaO}_{2}$-T90 (\%TST), percentage of total sleep time spent with arterial oxygen saturation less than $90 \%$.

unrelated to the interventions and three because of treatment intolerance.

Table 2 shows the polysomnography data from the end of treatment arm studies. Compared with placebo CPAP, therapeutic CPAP markedly reduced OSA severity. Table 3 shows the main treatment effect averaged over $24 \mathrm{~h}$ after placebo adjustment on coagulation markers.

FV, FVIII and vWF were significantly lower on CPAP than placebo (table 3 and figure 2). These differences were mainly evident during the sleep period (FV and FVIII) or after awakening (vWF). In contrast, fibrinogen, FVII, D-dimer and PAI-1 were not different between the two treatments (figure 3). In addition to treatment effects, figures 2 and 3 show that all coagulation markers displayed discrete diurnal variations $(p<0.05$ for all coagulation factor time effects) independent of treatment $(p>0.3$ for all analyses of time by treatment interactions).

\section{DISCUSSION}

Compared with placebo, 2 months' treatment of OSA with CPAP reduced FV and FVIII levels during the sleep period and vWF levels after awakening. In addition, the results demonstrate marked day-night variations that were independent of treatment in all the coagulation parameters (FV, FVII, FVIII, vWF,

Table 2 End of treatment polysomnography data

\begin{tabular}{llcc}
\hline Characteristic & $\begin{array}{l}\text { CPAP } \\
\text { (mean } \pm \text { SEM) }\end{array}$ & $\begin{array}{l}\text { Placebo } \\
\text { (mean } \pm \text { SEM) }\end{array}$ & p Value \\
\hline Sleep apnoea & & & \\
AHI (events/h) & $6.9 \pm 2.1$ & $40.0 \pm 5.0$ & $<0.00001$ \\
ODI (events/h) & $5.2 \pm 1.9$ & $38.7 \pm 4.1$ & $<0.00001$ \\
$\mathrm{SaO}_{2}-\mathrm{T90}(\% \mathrm{TST})$ & $1.0 \pm 0.4$ & $9.7 \pm 2.2$ & $<0.0005$ \\
$\mathrm{Min} \mathrm{SaO}_{2}(\%)$ & $89 \pm 1.4$ & $78 \pm 1.6$ & $<0.00001$ \\
Treatment compliance (h/night) & $4.4 \pm 2.2$ & $3.4 \pm 2.3$ & $<0.05$ \\
ESS & $7.6 \pm 0.8$ & $9.4 \pm 0.7$ & 0.1 \\
\hline
\end{tabular}

AHI, Apnoea Hypopnoea Index; CPAP, continuous positive airway pressure; ESS, Epworth sleepiness score; $\mathrm{Min} \mathrm{SaO}_{2}$, minimum arterial oxygen saturation; ODI, Oxygen Desaturation Index; $\mathrm{SaO}_{2}$-T90 (\%TST), percentage of total sleep time spent with arterial oxygen saturation less than $90 \%$.
Table 3 Main effects continuous positive airway pressure (CPAP) versus placebo

\begin{tabular}{lcccc}
\hline & $\begin{array}{l}\text { Main effects } \\
\text { CPAP-placebo } \\
\text { mean difference }\end{array}$ & $\begin{array}{l}\text { Lower } \\
\mathbf{9 5 \%} \text { Cl }\end{array}$ & $\begin{array}{l}\text { Upper } \\
\mathbf{9 5 \%} \text { Cl }\end{array}$ & p Value \\
\hline Coagulation marker & & & & \\
PAI-1 (U/ml) & 0.7 & -0.6 & 2.1 & 0.29 \\
vWF (\%) & -3.9 & -7.0 & -0.8 & 0.013 \\
Fibrinogen (g/litre) & -0.0 & -0.1 & 0.0 & 0.3 \\
D-dimer ( $\mu \mathrm{g} / \mathrm{ml})$ & -0.0 & -0.02 & 0.02 & 0.9 \\
FV (\%) & -4.2 & -6.6 & -1.8 & $<0.001$ \\
FVII (\%) & 0.50 & -1.37 & 2.36 & 0.6 \\
FVIII (\%) & -6.2 & -10.7 & -1.7 & 0.007 \\
\hline
\end{tabular}

FV, factor V; FVII, factor VII; FVIII, factor VIII; PAl-1, plasminogen activator inhibitor-1; VWF, von Willebrand Factor.

PAI-1, D-dimer and fibrinogen). The reduction in FV and FVIII during sleep with CPAP treatment may in part contribute to a lowering of cardiovascular risk, particularly in the period when there is a known increase in the prevalence of cardiovascular events in patients with OSA. ${ }^{20} 22$

Previous cross-sectional studies support increased coagulability and decreased fibrinolytic capacity in $\mathrm{OSA}^{9-12} 26$ with improvements following CPAP treatment. ${ }^{14} 23$ 26-29 However, the associations with OSA and changes with CPAP treatment have not been uniformly confirmed for all markers. ${ }^{14}{ }^{16}$ Furthermore, although OSA-associated sleep fragmentation and hypoxia have been linked to levels of some prothrombotic factors, ${ }^{9} 11$ the findings are not uniform, with several studies showing no association. ${ }^{23} 273031$ In the context of our own findings, although there are no studies that have examined FV in OSA, previous studies have examined vWF and FVIII but the findings have been inconsistent. In two cross-sectional studies that compared vWF in OSA patients with matched controls, one study showed elevated levels in OSA, ${ }^{26}$ while a second did not. ${ }^{32}$ Also, in a larger cross-sectional study of subjects without a history of sleep disorders, vWF levels were associated with sleep fragmentation but this was independent of OSA. ${ }^{9}$ Similarly, two randomised trials have found no change in vWF levels after CPAP versus placebo CPAP treatment. ${ }^{14} 16$ The latter study was the only study to assess FVIII in OSA and found no post-CPAP change. ${ }^{16}$

Interestingly, the patients from these randomised trials were of similar age and BMI and with a similar severity of OSA to the patients in our own study. Hence it is unclear why our results are at odds with these previous studies. One potential explanation may relate to sampling frequency. Previous CPAP intervention studies have only incorporated single samples which varied with respect to the time of day they were drawn and it is clear from our own data that all markers we assayed display a marked diurnal variability. Hence the lack of change in FVIII and vWF in the previous study after CPAP may have occurred because samples were taken later in the day between 11:00 and $13: 00,{ }^{16}$ which coincides with a period in our own study when we could not detect any treatment effect. A second potential explanation may relate to an inadequate length of OSA treatment which was only 2 weeks in one trial ${ }^{14}$ and 1 month in the other. ${ }^{16}$ In support of this, a previous uncontrolled study found that 2 months of CPAP treatment was associated with a trend $(p=0.07)$ for lower vWF levels. ${ }^{26}$ Finally, the difference in results may be due to differences in assay techniques.

In contrast to FV, FVIII and vWF, we found no effect of CPAP treatment versus placebo CPAP on FVII, PAI-1, fibrinogen or Ddimer and these results are somewhat consistent with other 
CPAP intervention studies. In particular, there are only two studies that have assessed the effects of CPAP treatment on FVII, with one study showing a gradual lowering but only after a 6-month period ${ }^{27}$ while a second 1 -month randomised trial failed to show any change. ${ }^{16}$ Decreased fibrinolytic activity resulting from increased levels of PAI-1 have been shown in patients with OSA $^{12} 1329$ but in contrast to our own study, a previous trial has shown a fall in levels after CPAP. ${ }^{14}$ Similarly, fibrinogen has also been found to be elevated in OSA, ${ }^{13} 3334$ however in keeping with our own results, two studies found no change after CPAP. ${ }^{16} 35$ Finally, although one study has linked the hypoxia of OSA with D-dimer, ${ }^{36}$ two recent studies have failed to find any association, ${ }^{9} 13$ and consistent with our own findings, CPAP treatment failed to change D-dimer levels. ${ }^{14}$

There are numerous experimental data implicating the haemostatic system as a determinant of both atherothrombosis and atherogenesis. ${ }^{37}$ vWF and FVIII are stored in endothelial cells and platelets and both have been used as surrogate markers of endothelial dysfunction. Elevations in these factors may play a direct role in atherosclerosis progression, ${ }^{37}$ giving rise to increased cardiovascular events of arterial and venous origin. Indeed, in the Northwick Park Heart Study, levels of FVIII were significantly higher in patients who died of cardiovascular disease than in those who survived. ${ }^{38} 39$ Studies also suggest that for each $10 \mathrm{IU} / \mathrm{dl}$ increment in FVIII (equivalent to $10 \%$ using our assay method), the risk for a single episode of venous thromboembolism (VTE) increases by 10\%, whereas for recurrent VTE this increases to $24 \%{ }^{40}$ Furthermore, high concentrations of vWF have been linked with recurrent MI and are involved in the setting of acute coronary syndromes. ${ }^{41}{ }^{42}$ Similarly, in the presence of hypertension, high levels of FV (>109\%) which shares a similar storage pathway to vWF has been associated with an increased risk for MI. ${ }^{43}$

It follows that similar mechanisms, involving hypertension, endothelial dysfunction and increased coagulation in OSA may be involved in increasing the risk of cardiovascular events. Although this is still unproven, OSA is known to be underdiagnosed in patients after acute $\mathrm{MI}^{44}$ and a recent study found a higher risk of OSA in patients diagnosed with acute pulmonary embolism, ${ }^{45}$ raising the possibility that OSA may be causally involved. ${ }^{46}$ It also follows that lower coagulability indicated by lower FV, FVIII and vWF either during sleep or in the post-awakening period after CPAP may partly signify a lowering of risk during this time. However, it is uncertain whether the small difference in coagulability with CPAP would translate to a reduction in risk as there are no extensive data to quantify the risk reduction that is attributable to reduced coagulability. We could also not demonstrate any difference in PAI-1, fibrinogen, D-dimer and FVII with CPAP compared with placebo despite an association between these factors and increased cardiovascular risk. ${ }^{47} 48$ Therefore we recognise that these CPAP effects, although partly supportive of reduced endothelial and platelet activation in our patients, do not necessarily translate to a reduction in event risk.

In addition to treatment-related effects, our study has demonstrated diurnal variations that were independent of treatment in most of the coagulation markers we assessed. The increase in PAI-1 and decrease in D-dimer during sleep are consistent with findings from a previous study. ${ }^{10}$ An increase in PAI-1 and fibrinogen occurred between 00:00 and 06:30, along with increases in vWF, FVIII, FVII and FV between 03:00 and 06:30. Together with a diurnal variation of platelet aggregation, ${ }^{49}$ these haemostatic rhythms likely play an important role in mediating increased risk for MI and stroke in the early morning period. ${ }^{17}$ As such, future studies are needed to further explore the mechanisms that drive these rhythms.

Finally, it is important to mention several aspects of our trial design which may limit the interpretation of our results. First, the inability to detect a change in some markers after CPAP treatment in our study may have occurred because of an insufficient treatment period. Alternatively, a carry-over effect may have occurred despite a 1-month washout period between treatments. Furthermore, without baseline data prior to each treatment we cannot determine whether the lower levels of some markers on CPAP were due to positive effects of CPAP or negative effects of the placebo device. This will require further study in larger trials that include longer treatment periods, preferably using a parallel design. Second, although we planned to assess coagulation markers in addition to our primary outcome of postprandial lipids, ${ }^{24}$ the selection of these markers was determined post hoc. Hence the study may have been underpowered to detect changes in some markers. Finally, although the lower concentration for some markers after CPAP compared with placebo is consistent with reduced endothelial and platelet activation, any cause-effect relationship on hard cardiovascular endpoints would require confirmation in prospective randomised longitudinal cohort studies.

\section{CONCLUSIONS}

This study has shown that CPAP treatment of OSA compared with placebo significantly reduces coagulability through a reduction in FV, FVIII and vWF. In addition, the study revealed diurnal variations in many coagulation markers. Future studies will need to explore the mechanisms that control the diurnal variation in haemostatic balance and whether this improves overall with long-term treatment of OSA. Although our findings suggest that CPAP may reduce cardiovascular risk in OSA through reducing coagulability, future prospective longitudinal studies are required to determine whether this reduces cardiovascular morbidity and mortality.

Contributors Professor Grunstein, Dr Phillips, Dr Yee and Dr Sullivan conceived the original experimental design from which these data have arisen. Professor Grunstein and Dr Phillips jointly obtained research funding from the National Health and Medical Research Council of Australia to conduct this 3-year trial. Professor Tofler, Mr McEwen (PhD candidate), Dr Morel-Kopp, Associate Professor Ward and Dr Phillips were responsible for conceiving which coagulation markers to examine from this study and $\mathrm{Mr}$ McEwen and Dr Morel-Kopp performed the assays. Dr Phillips performed all statistical analyses and wrote the original manuscript draft with Mr McEwen. All authors made significant contributions to the interpretation of data and revision of the manuscript for important intellectual content prior to submission.

Funding This research was supported through the National Health and Medical Research Project Grant 301936 (RRG) and Fellowship 571179 (CLP) and 1022730 (RRG). BJM received a scholarship through North Shore Heart Research Foundation.

Competing interests None to declare.

Ethics approval Ethics approval was provided by Sydney South West Area Health Service HREC.

Provenance and peer review Not commissioned; externally peer reviewed.

\section{REFERENCES}

1. Marshall NS, Wong KK, Liu PY, et al. Sleep apnea as an independent risk factor for all-cause mortality: the Busselton Health Study. Sleep 2008;31:1079-85.

2. Young T, Finn L, Peppard P, et al. Sleep disordered breathing and mortality: eighteen-year follow-up of the Wisconsin sleep cohort. Sleep 2008;31:1071-8.

3. Redline S, Yenokyan G, Gottlieb DJ, et al. Obstructive sleep apnea hypopnea and incident stroke: the Sleep Heart Health Study. Am J Respir Crit Care Med 2010;182:269-77.

4. Gottlieb DJ, Yenokyan G, Newman AB, et al. Prospective study of obstructive sleep apnea and incident coronary heart disease and heart failure: the Sleep Heart Health Study. Circulation 2010;122:352-60. 
5. Marin JM, Carrizo SJ, Vicente E, et al. Long-term cardiovascular outcomes in men with obstructive sleep apnoea-hypopnoea with or without treatment with continuous positive airway pressure: an observational study. Lancet 2005;365:1046-53.

6. Bazzano LA, Khan Z, Reynolds K, et al. Effect of nocturnal nasal continuous positive airway pressure on blood pressure in obstructive sleep apnea. Hypertension 2007:50:417-23.

7. Cross MD, Mills NL, Al-abri M, et al. Continuous positive airway pressure improves vascular function in obstructive sleep apnea hypopnea syndrome: a randomized controlled trial. Thorax 2008;63:578-83.

8. Jelic S, Padeletti M, Kawut SM, et al. Inflammation, oxidative stress, and repair capacity of the vascular endothelium in obstructive sleep apnea. Circulation 2008;117:2270-8.

9. von Känel R, Loredo JS, Ancoli-lsrael S, et al. Association between polysomnographic measures of disrupted sleep and prothrombotic factors. Chest 2007:131:733-9.

10. von Kanel R, Natarajan L, Ancoli-Israel S, et al. Day/night rhythm of hemostatic factors in obstructive sleep apnea. Sleep 2010;33:371-7.

11. Terada S, Koyama $\mathrm{T}$, Watanabe $\mathrm{H}$, et al. Abnormal coagulation and platelet profile in patients with obstructive sleep apnea syndrome. Int J Cardiol 2011;146:423-5.

12. Zamarrón C, Ricoy J, Riveiro A, et al. Plasminogen activator inhibitor-1 in obstructive sleep apnea patients with and without hypertension. Lung 2008;186:151-6.

13. Mehra R, Xu F, Babineau DC, et al. Sleep disordered breathing and pro-thrombotic biomarkers: cross-sectional results of the Cleveland Family Study. Am J Respir Crit Care Med 2010;182:826-33.

14. von Känel R, Loredo J, Ancoli-Israel S, et al. Association between sleep apnea severity and blood coagulability: treatment effects of nasal continuous positive airway pressure. Sleep Breath 2006;10:139-46

15. Guardiola JJ, Matheson PJ, Clavijo LC, et al. Hypercoagulability in patients with obstructive sleep apnea. Sleep Med 2001;2:517-23

16. Robinson GV, Pepperell JC, Segal HC, et al. Circulating cardiovascular risk factors in obstructive sleep apnoea: data from randomised controlled trials. Thorax 2004;59:777-82.

17. Haus E. Chronobiology of hemostasis and inferences for the chronotherapy of coagulation disorders and thrombosis prevention. Adv Drug Deliv Rev 2007:59:966-84.

18. Muller JE, Tofler GH, Stone PH. Circadian variation and triggers of onset of acute cardiovascular disease. Circulation 1989;79:733-43.

19. Goldberg RJ, Brady P, Muller JE, et al. Time of onset of symptoms of acute myocardial infarction. Am J Cardiol 1990;66:140-4.

20. Gami AS, Howard DE, Olson EJ, et al. Day-night pattern of sudden death in obstructive sleep apnea. N Engl J Med 2005;352:1206-14.

21. Willich SN, Levy D, Rocco MB, et al. Circadian variation in the incidence of sudden cardiac death in the Framingham Heart Study population. Am J Cardiol 1987;60:801-6.

22. Sert-Kuniyoshi FH, Garcia-Touchard A, Gami AS, et al. Day-night variation of acute myocardial infarction in obstructive sleep apnea. J Am Coll Cardiol 2008;52:343-6.

23. Bokinsky G, Miller M, Ault K, et al. Spontaneous platelet activation and aggregation during obstructive sleep apnea and its response to therapy with nasal continuous positive airway pressure. Chest 1995;108:625-30.

24. Phillips CL, Yee BJ, Marshall NS, et al. Continuous positive airway pressure reduces postprandial lipidemia in obstructive sleep apnea: a randomised, placebo controlled crossover trial. Am J Respir Crit Care Med 2011;184:355-61.

25. Anon. Sleep-related breathing disorders in adults: recommendations for syndrome definition and measurement techniques in clinical research. The Report of an American Academy of Sleep Medicine Task Force [see comment]. Sleep 1999:22:667-89.

26. El Solh A, Akinnusi M, Berim I, et al. Hemostatic implications of endothelial cell apoptosis in obstructive sleep apnea. Sleep Breath 2008;12:331-7.
27. Chin K, Kita $\mathrm{H}$, Noguchi $\mathrm{T}$, et al. Improvement of factor VII clotting activity following long-term NCPAP treatment in obstructive sleep apnoea syndrome. OJM 1998:91:627-33

28. Chin $\mathbf{K}$, Ohi $\mathrm{M}$, Kita $\mathrm{H}$, et al. Effects of NCPAP therapy on fibrinogen levels in obstructive sleep apnea syndrome. Am J Respir Crit Care Med 1996;153:1972-6.

29. Rangemark C, Hedner JA, Carlson JT, et al. Platelet function and fibrinolytic activity in hypertensive and normotensive sleep apnea patients. Sleep 1995;18:188-94.

30. Sanner BM, Konermann M, Tepel M, et al. Platelet function in patients with obstructive sleep apnoea syndrome. Eur Respir J 2000;16:648-52.

31. von Kanel R, Le DT, Nelesen RA, et al. The hypercoagulable state in sleep apnea is related to comorbid hypertension. J Hypertens 2001;19:1445-51.

32. Zamarrón-Sanz C, Ricoy-Galbaldon J, Gude-Sampedro F, et al. Plasma levels of vascular endothelial markers in obstructive sleep apnea. Arch Med Res 2006;37:552-5.

33. Peled N, Kassirer M, Kramer MR, et al. Increased erythrocyte adhesiveness and aggregation in obstructive sleep apnea syndrome. Thromb Res 2008;121:631-6.

34. Wessendorf TE, Thilmann AF, Wang YM, et al. Fibrinogen levels and obstructive sleep apnea in ischemic stroke. Am J Respir Crit Care Med 2000;162:2039-42.

35. Dorkova Z, Petrasova D, Molcanyiova A, et al. Effects of continuous positive airway pressure on cardiovascular risk profile in patients with severe obstructive sleep apnea and metabolic syndrome. Chest 2008;134:686-92.

36. Shitrit D, Peled N, Shitrit AB, et al. An association between oxygen desaturation and D-dimer in patients with obstructive sleep apnea syndrome. Thromb Haemost 2005:94:544-7.

37. Borissoff JI, Spronk HM, ten Cate H. The hemostatic system as a modulator of atherosclerosis. N Engl J Med 2011;364:1746-60.

38. Meade TW, Brozovic M, Chakrabarti RR, et al. Haemostatic function and ischaemic heart disease: principal results of the Northwick Park Heart Study. Lancet 1986;328:533-7

39. Meade TW, North WR, Chakrabarti R, et al. Haemostatic function and cardiovascular death: early results of a prospective study. Lancet 1980;1:1050-4.

40. Kraaijenhagen RA, in't Anker PS, Koopman MM, et al. High plasma concentration of factor VIIIC is a major risk factor for venous thromboembolism. Thromb Haemos 2000;83:5-9.

41. Jansson $\mathbf{J H}$, Nilsson TK, Johnson 0. von Willebrand factor in plasma: a novel risk factor for recurrent myocardial infarction and death. Br Heart $J$ 1991;66:351-5.

42. Spiel AO, Gilbert JC, Jilma B. von Willebrand factor in cardiovascular disease: focus on acute coronary syndromes. Circulation 2008;117:1449-59.

43. Redondo M, Watzke HH, Stucki B, et al. Coagulation factors II, V, VII, and X, prothrombin gene $20210 \mathrm{G} \rightarrow \mathrm{A}$ transition, and factor $\mathrm{V}$ Leiden in coronary artery disease: high factor $\mathrm{V}$ clotting activity is an independent risk factor for myocardial infarction. Arterioscler Thromb Vasc Biol 1999;19:1020-5.

44. Konecny T, Sert Kuniyoshi FH, Orban M, et al. Under-diagnosis of sleep apnea in patients after acute myocardial infarction. J Am Coll Cardiol 2010;56:742-3.

45. Epstein MD, Segal LN, Ibrahim SM, et al. Snoring and the risk of obstructive sleep apnea in patients with pulmonary embolism. Sleep 2010;33:1069-74.

46. Cistulli PA, Phillips CL. Expanding the clinical spectrum of OSA -an association with pulmonary embolism? Sleep 2010;33:1009-10.

47. Hamsten A, de Faire U, Walldius G, et al. Plasminogen activator inhibitor in plasma: risk factor for recurrent myocardial infarction. Lancet 1987:2:3-9.

48. Scarabin PY, Aillaud MF, Amouyel P, et al. Associations of fibrinogen, factor VII and PAl-1 with baseline findings among 10,500 male participants in a prospective study of myocardial infarction - the PRIME Study. Prospective Epidemiological Study of Myocardial Infarction. Thromb Haemost 1998;80:749-56.

49. Tofler GH, Brezinski D, Schafer Al, et al. Concurrent morning increase in platelet aggregability and the risk of myocardial infarction and sudden cardiac death. $N$ Engl J Med 1987;316:1514-18. 